\title{
La place de l'adjectif épithète en français : ce que nous apprennent les corpus oraux ${ }^{1}$
}

\author{
Benzitoun, Christophe \\ Université de Lorraine \& CNRS ATILF \\ Christophe.Benzitoun@univ-lorraine.fr
}

\section{Préambule}

Ce travail s'inscrit dans le cadre du projet Français Contemporain Vernaculaire (FRACOV) dont l'objectif est l'élaboration de fiches synthétiques portant sur la grammaire de la variété vernaculaire du français. Le projet FRACOV est mené sous l'égide de l'Institut de Linguistique Française (ILF, FR2393) et a pour responsables Pierre Larrivée (Université de Caen) et Florence Lefeuvre (Université Paris 3). Les fiches contenant les descriptions grammaticales seront mises en ligne sur une plateforme collaborative en accès libre. Chaque fiche contiendra la description d'un phénomène linguistique en se centrant sur des données « vernaculaires » plutôt que sur la théorie. Le contenu des fiches devra être accessible à un large public intéressé par la langue et pas aux seuls spécialistes, ce qui explique que la rédaction soit volontairement assez peu technique.

Dans le présent article, nous présentons la fiche que nous avons élaborée dans le cadre de ce projet et qui porte sur la place de l'adjectif épithète en français parlé. Cette fiche est en phase de finalisation et profitera des retours de la communauté de la linguistique française au Congrès Mondial de Linguistique Française. Nous y avons adjoint une partie sur les études antérieures, partie qui ne se trouvera pas dans la fiche finale. En effet, le projet FRACOV n'a pas de visée encyclopédique. Il ne s'agit pas de faire la synthèse de l'ensemble des publications portant sur un point particulier de la grammaire du français. Toutefois, l'adjonction d'une courte section « état de la question » nous paraissait incontournable dans le cadre d'une publication scientifique.

Ci-dessous, nous commençons par proposer une définition de ce que l'on appelle traditionnellement les adjectifs épithètes (section 1). Puis nous détaillons brièvement le problème principal traité dans cet article (section 2). La section 3 contient une sélection des principales publications sur la question. Dans la section 4, nous exposons les tendances générales observables dans les corpus de français parlé. Dans la section 5, nous détaillons les deux principaux types d'alternance complexe, à savoir les alternances en distribution complémentaire et les alternances plus difficiles à décrire pour lesquelles de plus amples investigations seront nécessaires. Dans la section 6, nous proposons une classification des adjectifs en fonction de la place qu'ils occupent auprès du substantif dans les corpus que nous avons consultés et de la fréquence respective de chacune des places. La section 7 est une synthèse des principaux fonctionnements observés.

\section{Définitions}

L'adjectif est une classe de mots difficile à circonscrire, tant en linguistique française (Goes, 1999 ; Noailly, 1999) qu'en linguistique générale (Creissels, 2006 : chapitre 12). Pour le français, de nombreuses grammaires invoquent principalement deux propriétés morphosyntaxiques :

- l'adjectif s'accorde en genre et en nombre avec le substantif auquel il se rapporte ;

- on peut lui adjoindre l'adverbe très.

Mais il a été démontré depuis longtemps que ces propriétés ne sont pas discriminantes et qu'elles ne s'appliquent pas à l'ensemble des unités que l'on considère traditionnellement comme des adjectifs. En français parlé, le genre et le nombre sont rarement audibles (sauf contextes de liaison et quelques formes 
telles égal/égaux, grand/grande, etc.), ce qui rend inopérante la partie morphologique de la définition. De plus, seuls certains adjectifs acceptent la modification à l'aide de très (un garçon très petit vs *l'année très dernière). En fait, aucun critère distributionnel ou morphologique ne permet de poser une séparation claire entre les substantifs et les adjectifs si bien que l'on peut être amené à y voir un continuum (Noailly, 1999).

Le terme "épithète», quant à lui, renvoie à la fonction de l'adjectif, à savoir celle de dépendre directement d'un substantif à l'intérieur d'un syntagme nominal. Un adjectif épithète est donc un mot qui dépend directement d'un substantif et qui, dans certaines cas, restreint l'extension de ce substantif. Les éléments en gras dans les exemples suivants sont des adjectifs épithètes :

1) voilà alors je disais donc que la sculpture c'était ma grande passion [crfp ; pri-per-2]

2) donc euh l'année prochaine normalement je devrais retravailler [crfp ; pri-pne-1]

L'adjectif épithète peut se placer après ou avant le substantif auquel il se rapporte. Plusieurs adjectifs peuvent se rapporter à un même substantif :

3) tandis que lui il a vous lirez ça dans l'introduction il est un obscur petit dijonnais qui n'a pas pu trouver sa place dans le Cénacle romantique [corpaix ; poésie]

Les adjectifs peuvent être mobiles ou bien avoir une place fixe. Les adjectifs pouvant se trouver avant et après le substantif seront qualifiés d'adjectifs «alternants ». La qualification d'adjectif alternant sera attribuée exclusivement aux adjectifs pour lesquels on trouve au moins une occurrence dans chacune des deux places autour du substantif dans les corpus que nous avons consultés.

\section{Situation du problème}

Le problème principal que l'on rencontre quand on s'intéresse à la place de l'adjectif épithète en français est celui de savoir si une place donnée pour un adjectif donné est motivée et s'il existe des blocages liés à des paramètres observables. Dit autrement, observe-t-on des propriétés linguistiques (morphosyntaxiques, sémantiques ou autres) permettant de prédire la place d'un adjectif ou un blocage éventuel ?

Pour les deux exemples d'ancien ci-dessous, il est clair que les deux places sont attachées à des sens différents :

4) alors le musée des Beaux-Arts euh est installé dans des bâtiments anciens [crfp ; pub-orl-1]

5) soit on surélevait euh le petit local le petit local entre parenthèses c'étaient les anciens bains douches de La Destrousse [corpaix ; médiathèque]

En effet, des bâtiments anciens, ce sont toujours des bâtiments mais construits à une époque lointaine, alors que les anciens bains douches ne sont plus des bains douches.

De même, différents paramètres morphosyntaxiques peuvent bloquer un adjectif dans une place. C'est le cas par exemple du déterminant indéfini dans l'exemple suivant :

6) elle est venue comme ça un petit peu sans vraiment euh enfin sans avoir une gro- une formation principale arts dramatiques $[\mathrm{pfc} ; 75 \mathrm{ccm} 1]$

Il serait en effet difficile de produire une principale formation alors que la principale formation ne pose aucun problème.

Toute la difficulté est donc d'essayer de mettre en lumière les règles régissant la place de l'adjectif épithète, ce que de nombreux auteurs ont tenté par le passé, mais sans parvenir à produire une théorie générale, comme nous allons le voir dans la partie suivante. 


\section{Etudes antérieures}

La place de l'adjectif épithète par rapport au substantif auquel il se rapporte est un sujet qui a donné lieu à une littérature conséquente en linguistique française. En guise de sélection réduite - et en partie arbitraire - on peut citer au moins trois articles de synthèse (Delomier, 1980 ; Wunderli, 1987 ; Forsgren, 1997) et plusieurs ouvrages dont Blinkenberg (1928 \& 1933), Reiner (1968), Forsgren (1978), Larsson (1994) et Wilmet (1986). On peut également mentionner la grammaire de Riegel et al. (2004) qui présente une vaste description du phénomène accessible à un large public dans sa section sur les adjectifs ainsi que le travail de NVlke (1996) sur la pertinence de prendre en compte la focalisation comme paramètre explicatif. A partir de l'ensemble de ces études, on peut dresser le bilan suivant, prenant la forme d'un constat d'échec dans la recherche d'une théorie globale :

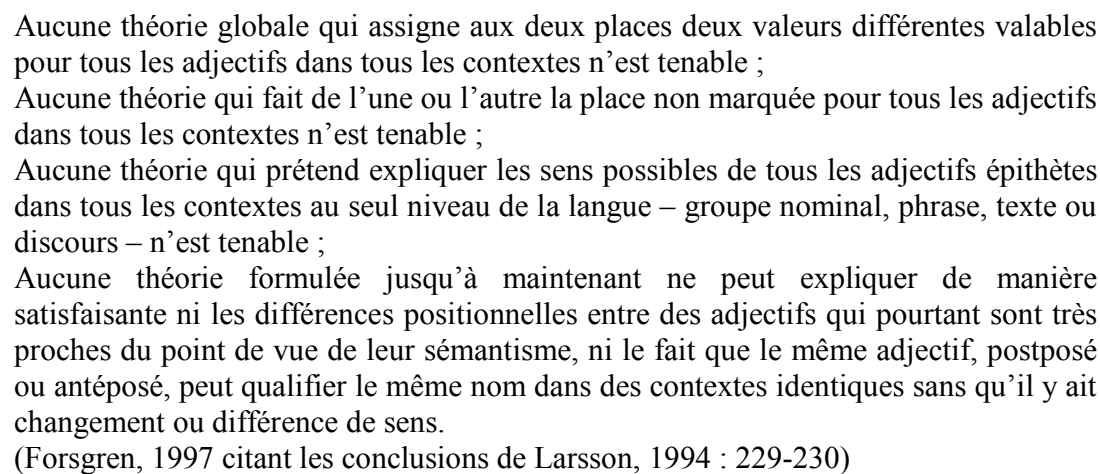

Toutefois, la quasi-totalité des publications portent sur des exemples écrits (majoritairement extraits de la presse et de la littérature) ou sur des exemples obtenus par introspection. Or, la nature et le « genre » des données prises en compte ont une incidence sur l'éventail des possibilités des unités linguistiques observées. Nous verrons que l'exploitation de données orales nous permet d'explorer de nouvelles pistes prometteuses et ainsi d'entrevoir d'autres régularités que celles mises au jour jusqu'à présent. La dimension du genre textuel a donc une place importante dans le présent travail, qui n'a pas pour ambition de dépeindre le fonctionnement des adjectifs en français mais principalement en français parlé non planifié. Nous nous démarquons donc clairement de travaux tels ceux de Cinque (1994), Bouchard (2002) et Knittel (2005), qui sont extrêmement poussés du point de vue de la finesse de la théorisation mais dont les travaux reposent sur des données construites plutôt que sur des corpus de productions réelles. Nous envisageons dans un travail ultérieur la confrontation entre nos résultats et ces travaux de théorisation afin d'améliorer la classification. Mais nous pouvons d'ores et déjà mentionner le fait que les approches sur corpus et les approches génératives sont si différentes que la nature même des questions posées et des phénomènes observés induit des résultats assez éloignés.

Par ailleurs, eu égard à l'importante variation des usages observables dans les corpus, les travaux actuels s'orientent plutôt vers l'étude des tendances (ou contraintes préférentielles) et non des règles systématiques (contraintes catégoriques). Voici quelques-unes des principales contraintes préférentielles permettant de rendre compte de la place des adjectifs, mises en évidence dans les études antérieures : sens de l'adjectif (un pauvre gars vs un gars pauvre), nombre grammatical (la semaine prochaine vs les prochaines semaines), présence d'autres adjectifs (une très grande bibliothèque vs une belle bibliothèque très grande), présence d'un modifieur pré-adjectival (une belle véranda vs une véranda très belle), substantif constructeur (un lien fort vs un fort taux d'humidité; un monde meilleur vs une meilleure organisation), emplois spécifiques et figements (une langue première vs une première étape), coordination avec un autre adjectif (une belle et difficile épreuve vs une épreuve difficile), forme du déterminant (cet étonnante discussion vs une discussion étonnante), nombre de syllabes (une très grande librairie vs une librairie extrêmement grande; une belle librairie vs une table magnifique), etc. Thuilier (2012) montre, à partir d'une étude statistique précise, que la plupart des contraintes préférentielles ont une influence et qu'il est possible de prédire la place des adjectifs en utilisant ces contraintes (avec un 
taux d'erreurs minime). La présence d'un modifieur post-adjectival, quant à elle, est une des seules contraintes catégoriques relevée jusqu'à présent :

7) si vous allez faire un petit tour sur les quais vous verrez qu'il y a des égouts des tuyaux gros comme ça tous les dix mètres [corpaix ; cours]

8) *vous verrez qu'il y a des gros comme ça tuyaux tous les dix mètres

Les difficultés rencontrées pour percevoir la régularité du système viennent en partie de la très grande variabilité avec laquelle les francophones (et plus particulièrement les écrivains et les journalistes) utilisent les adjectifs à l'écrit. Rares sont les adjectifs pour lesquels on ne trouve aucune attestation des deux places, y compris pour les relationnels (c'est-à-dire les adjectifs dérivés de substantifs) :

9) Mais, insoucieux de la solidarité gouvernementale, les communistes raniment le Mouvement de la paix, de stalinienne mémoire [L'Express, cité par Forsgren (1997)]

ou les adjectifs proches de participes passés :

\section{0) Mourir, il le voulait, il en avait l'enragée impatience. [E. Zola, La Débâcle]}

pourtant réputés impossibles à antéposer. Quelques linguistes partent même du principe que tous les adjectifs sont mobiles. Goes (1999) considère d'ailleurs que la mobilité représente un critère définitoire des adjectifs.

Contrairement aux études sur la langue écrite, celles portant sur le français parlé sont peu nombreuses. Il y a tout de même Blasco-Dulbecco \& Cappeau (2004; 2005), Fox (2012) (dans une perspective d'acquisition), Thuilier (2013), Benzitoun et al. (2010) et Benzitoun (2013), mais aucune étude d'envergure n'a pour l'instant été menée, ce qui fait que le présent article ne sera pas une synthèse de travaux antérieurs mais une description en partie inédite.

\section{Tendances générales en français parlé}

Comme nous l'avons vu ci-dessus, l'étude des données écrites et/ou obtenues par introspection mène à la conclusion que la totalité des adjectifs sont potentiellement alternants. Cependant, le fait qu'il s'agisse de données ayant un fort degré de planification a sans doute un impact non négligeable sur ce constat. Or, les données orales donnent accès aux « faits majeurs de la distribution, qui [sont] ainsi moins "parasités" par des phénomènes d'ordre stylistique (et donc plus atypiques)» (Cappeau, 2002:11), d'autant plus si le degré de planification du discours est réduit. L'usage des adjectifs étant notoirement influencé par des facteurs stylistiques, particulièrement dans les œuvres littéraires ${ }^{2}$, l'analyse de l'oral va donner à voir une autre organisation notamment par l'intermédiaire de la notion de "place préférentielle ». Nous illustrons les différences principales entre oral non planifié et écrit planifié un peu plus loin.

La ressource orale sur laquelle nous nous appuyons fait environ 2.300.000 mots. Elle est composée des corpus suivants : Corpus de Français Parlé Parisien ${ }^{3}-$ CFPP (Branca-Rosoff et al., 2012), Corpaix (Blanche-Benveniste, 1999), Corpus de Référence du Français Parlé - CRFP (Equipe DELIC, 2004), CORAL-ROM (Cresti \& Moneglia, 2005), Phonologie du Français Contemporain ${ }^{4}-$ PFC (Durand et al., 2002 ; Durand et al., 2005), Choix de Textes en Français Parlé - CTFP (Blanche-Benveniste et al., 2002).

Toutefois, afin de mettre en lumière les différences entre usage oral et écrit de la langue française, nous avons fait le choix d'utiliser un corpus de comparaison composé d'articles de presse. Pour ce faire, nous avons regroupé des corpus de presse, à savoir le corpus Chambers-Rostand (composé d'articles extraits des quotidiens L'Humanité, Le Monde et La Dépêche du Midi), une journée du corpus de l'Est Républicain et la partie presse du Corpus Evolutif de Référence du Français ${ }^{5}(C E R F)$. Ce corpus de comparaison représentant la presse écrite possède une taille comparable à celle de la partie orale.

Dans la ressources orale, nous avons relevé 33.397 occurrences d'adjectifs épithètes se répartissant de la manière suivante: 16.037 en antéposition (soit 48\%) et 17.360 en postposition (soit 52\%). En 
comparaison, Thuilier (2012) a relevé $27 \%$ d'antéposition et $73 \%$ de postposition dans le journal $L e$ Monde. Il existe donc des variations diamésiques ${ }^{6}$ importantes. Mais ces chiffres bruts masquent de très grandes disparités. Dans un premier temps, il est primordial de distinguer les adjectifs alternants afin d'avoir une vision plus précise :

\begin{tabular}{|l|c|c|c|c|}
\hline & Antéposé & Postposé & Alternant & Total \\
\hline \multirow{2}{*}{ Lemmes } & 41 & 1943 & 162 & 2146 \\
& $(1,9 \%)$ & $(90,6 \%)$ & $(7,5 \%)$ & $(100 \%)$ \\
\hline \multirow{2}{*}{ Occurrences } & 624 & 13643 & 19130 & 33397 \\
& $(1,9 \%)$ & $(40,8 \%)$ & $(57,3 \%)$ & $(100 \%)$ \\
\hline Ratio occ./lemmes & 15,2 & 7 & 118,1 & 15,6 \\
\hline
\end{tabular}

Tableau 1. Répartition générale des adjectifs à l'oral

Ce tableau met en évidence des tendances très nettes : un tout petit nombre d'adjectifs uniquement antéposés, une très large majorité d'adjectifs uniquement postposés et un faible nombre d'adjectifs alternants. A partir des comptages effectués par Wilmet (1980), sur un corpus pourtant deux fois moins volumineux, on constate que les adjectifs épithètes sont comparativement beaucoup plus fréquents (29.016 occurrences), plus variés (3.835 lemmes différents) et que le nombre d'adjectifs alternants est nettement plus important (645 lemmes différents) dans les œuvres littéraires contemporaines. Et on peut faire un constat similaire à partir de textes journalistiques.

En ce qui concerne spécifiquement les adjectifs alternants, la diversité limitée des formes à l'oral s'accompagne d'un nombre d'occurrences extrêmement élevé. Et si l'on regarde de manière encore plus précise, on s'aperçoit que près des $4 / 5^{\text {ème }}$ des adjectifs alternants sont antéposés. Cela s'explique par le fait que dans la classe des adjectifs alternants se trouvent les adjectifs épithètes les plus fréquents (par ordre de fréquence décroissante observée dans la ressource orale) : petit, autre, grand, même, premier, bon, gros, certain, dernier, beau. Or ce sont justement des adjectifs qui s'antéposent à une très large majorité.

\section{Les deux principaux types d'alternance complexe}

\subsection{Alternances en distribution complémentaire}

En plus des adjectifs qui n'ont qu'un sens et un fonctionnement positionnel limité et relativement facile à circonscrire (ce que nous montrons plus loin), il en existe d'autres ayant plusieurs sens et des fonctionnements diversifiés. Les exemples de ce type tendent vers la distribution complémentaire, c'est-àdire vers une répartition positionnellement distincte de chacun des usages. C'est le cas par exemple de sale. Quand il est antéposé, il est synonyme de « désagréable » :

11) quand je suis arrivé là-bas un peu le sale gosse de la rive gauche dont la grand-mère avait refait des blousons dans les vieux vestons du grand-père etc. [crfp ; pri-rou-2]

Quand il est postposé, il est antonyme de «propre» :

12) c'est que c'est un quartier sale par rapport à la rue Barbet de Jouy qui était propre [cfpp ; Audin_ $7^{\mathrm{e}}$ ]

L'adjectif prochain possède également une distribution régulière - quoique plus complexe à décrire - à l'oral non planifié ${ }^{7}$. Lorsqu'il est postposé, il porte exclusivement sur des substantifs à sémantisme temporel au singulier. Dans ce contexte, il doit être accompagné d'un déterminant défini ou ne comporter aucun déterminant. Ainsi, on trouve des exemples tels l'année prochaine, la semaine prochaine, lundi prochain mais pas l'événement prochain ou une année prochaine. De même, nous n'avons trouvé aucun 
exemple où prochain est postposé et au pluriel (du type les années prochaines), quelle que soit la classe sémantique du substantif. Il y a donc une corrélation stricte entre la classe sémantique du substantif (temporel), la postposition, le nombre grammatical (singulier) et la nature du déterminant (défini). Le seul substantif qui enfreint cette régularité est fois, avec lequel prochain s'antépose très majoritairement (la prochaine fois est plus courant que la fois prochaine). Il peut également être précédé d'un déterminant indéfini (une prochaine fois). Ce fonctionnement particulier est sans doute lié au fait que fois ne délimite pas un empan temporel clairement défini, contrairement à semaine ou mois.

En plus de fois, toutes sortes de substantifs peuvent se rencontrer lorsque prochain est antéposé, au singulier comme au pluriel :

13) alors ma prochaine destination je crois que c'est le nord de la France ou plus précisément l'est les régions froides [crfp ; pri-val-2]

14) eh bien on va accueillir maintenant nos nos prochaines invitées [coralrom ; fmedts05]

En ce qui concerne les substantifs temporels, nous avons observé qu'une des deux conditions suivantes devaient être remplies pour que prochain puisse s'antéposer. Soit le substantif est au pluriel :

15) nous on vend essentiellement de la matière grise c'est-à-dire de la réflexion sur euh sur l'organisation et sur la stratégie que peut avoir l'entreprise euh dans les prochaines années [crfp ; pro-pcr-1]

soit il comporte un complément :

16) ma prochaine semaine de vacances je ne sais même pas quand la prendre [cfpp; duchemin_ceret_11e]

Toutefois, l'ensemble des régularités décrites ci-dessus ne s'observent pas dans les écrits planifiés. Dans un corpus écrit diversifié ${ }^{8}$ de taille identique à la ressource orale que nous utilisons, nous avons trouvé les exemples suivants, qui enfreignent tous une des régularités vues ci-dessus.

- Postposition à un substantif autre que temporel :

17) le voyageur se hâte vers la station prochaine [cerf, roman]

- Postposition à un substantif temporel au pluriel :

18) qui doivent s'être arrêtés dans les mois prochains [cerf, chirac]

- Postposition à un substantif autre que temporel précédé d'un déterminant indéfini :

19) Il annonce une normalisation prochaine de la situation [cerf, le monde]

Et nous observons une tendance identique en ce qui concerne les données obtenues par introspection. Berthonneau (2002) invente notamment l'exemple ci-dessous, qu'elle considère comme étant acceptable :

20) Le maire prochain devra mettre un terme à ces pratiques.

À la régularité observée pour les données orales se substitue donc un placement plus libre dans les productions écrites et donc une organisation moins visible. Ainsi, il y aurait une tendance de l'écrit à enfreindre le fonctionnement régulier utilisé à l'oral. Cela explique sans doute, au moins en partie, les difficultés que les chercheurs ont éprouvées jusqu'à présent pour élaborer une théorie globale du placement des adjectifs en français et illustre bien l'influence de facteurs stylistiques beaucoup plus présente dans les corpus écrits observés qu'à l'oral non planifié.

Pour résumer, voici les résultats tirés de Benzitoun et al. (2010) : 


\begin{tabular}{|c|c|c|c|}
\hline \multicolumn{2}{|c|}{ Oral (164 occ.) } & \multicolumn{2}{|c|}{ Écrit (290 occ.) } \\
\hline \multicolumn{2}{|c|}{ Singulier (149 occ.) } & \multicolumn{2}{|c|}{ Singulier (217 occ.) } \\
\hline Antéposé (51 occ.) & Postposé (98 occ.) & Antéposé (95 occ.) & Postposé (122 occ.) \\
\hline $\begin{array}{l}\text { Noms temporels }(51 \%) \\
\text { fois }(25 \text { occ. } / 26)\end{array}$ & $\begin{array}{l}\text { Noms temporels }(100 \%) \\
\text { annéelan ( } 48 \text { occ.) } \\
\text { semaine }(23 \text { occ.) }\end{array}$ & $\begin{array}{l}\text { Noms temporels }(20 \%) \\
\text { fois }(12 \text { occ. } / 19) \\
\text { siècle }(6 \text { occ. })\end{array}$ & $\begin{array}{l}\text { Noms temporels }(86,1 \%) \\
\text { annéelan (31 occ.) }\end{array}$ \\
\hline \multirow[t]{2}{*}{$\begin{array}{l}\text { Autres noms }(49 \%) \\
\text { disque, débat, etc. }\end{array}$} & \multirow[t]{2}{*}{ Autres noms $(0 \%)$} & \multirow[t]{2}{*}{ Autres noms $(80 \%)$} & $\begin{array}{l}\text { Sens de « proche » }(9 \%) \\
\text { victoire, mort, etc. }\end{array}$ \\
\hline & & & Autres noms $(4,9 \%)$ \\
\hline \multicolumn{2}{|c|}{ Pluriel (15 occ.) } & \multicolumn{2}{|c|}{$\begin{array}{l}\text { Pluriel (73 occ.) } \\
\end{array}$} \\
\hline Antéposé (15 occ.) & Postposé ( 0 occ.) & Antéposé (64 occ.) & Postposé (9 occ.) \\
\hline \multirow[t]{2}{*}{ années, vacances, etc. } & & Noms temporels $(62,5 \%)$ & \multirow[t]{2}{*}{ mois, intimités } \\
\hline & & Autres noms $(37,5 \%)$ & \\
\hline
\end{tabular}

Tableau 2. Fréquences comparées de l'adjectif prochain

À la lumière de l'exemple de prochain, on voit bien que la fréquence relative à chaque place n'est pas forcément pertinente. Se limiter ici à dire que l'on trouve, en français parlé, prochain 66 fois en antéposition et 98 fois en postposition n'est guère suffisant. Cela ne permet pas de rendre compte du fonctionnement de cet adjectif.

Cependant, il s'agit là d'un cas peu fréquent dans nos données. En effet, les alternances régulières complexes de ce type concernent relativement peu d'adjectifs en français parlé comparées aux adjectifs se limitant à une unique place ou se distinguant par le sens (voir ci-dessous). Il existe également d'autres adjectifs alternants, peu nombreux, pour lesquels des investigations plus poussées seront nécessaires. Nous illustrons ce phénomène dans la partie suivante.

\subsection{Alternances demandant un travail complémentaire}

S'il est possible de mettre en évidence que la plupart des adjectifs alternants possèdent un fonctionnement régulier (place corrélée à un sens ou bien à un contexte morphosyntaxique spécifique), certains ont une régularité plus difficile à circonscrire. Pour nouveau, par exemple, il y a une corrélation partielle entre la place et le sens. En postposition, on ne trouve que des occurrences ayant le sens de "nouveauté/ innovation $»$ :

21) en fait on voit de plus en plus de mots nouveaux qui apparaît \{sic\} chaque année dans les dictionnaires [corpaix ; 12fran]

En antéposition, on peut tout aussi bien trouver des exemples ayant ce même sens :

22) c'est peut-être pas seulement pour ça c'est pour nous apprendre euh peut-être de nouveaux mots euh de nouvelles structures [corpaix ; 19poème]

ou bien des occurrences dont le sens a trait à l’ « itération » (comme prochain et dernier) :

23) il ne peut supporter qu'une nouvelle fois des obstacles empêchent le libre cours de son ique-de son existence [coralrom ; fnatla02]

On trouve même des exemples où les deux places sont occupées :

24) en revanche je vois sur cette mission il y a deux nouveaux consultants tout nouveaux nouveaux dont c'est la première mission [crfp ; pro-pcr-1]

Ainsi, il y a deux sens plus ou moins faciles à distinguer et l'interprétation ne repose pas systématiquement sur une différence de place. C'est le substantif dont dépend nouveau ou bien la prise en compte d'un contexte plus large qui permet de circonscrire le sens. Il faut donc recourir à l'observation de 
paramètres contextuels divers pour modéliser le fonctionnement de cet adjectif. En tout cas, la place, le substantif ou une propriété morphosyntaxique (nature du déterminant, présence d'un adverbe modifieur, etc.) ne permettent pas à eux seuls de calculer son sens. De plus amples investigations seront nécessaires pour parvenir à comprendre son fonctionnement.

Pour l'adjectif fort, certaines occurrences sont bloquées en antéposition (25), d'autres en postposition (26) et d'autres encore sont mobiles ((27) et (28)) :

25) en plus de ça il y avait un fort taux d'humidité [coralrom ; fmedrp01]

26) donc afin de souligner les points forts et de repérer les faiblesses et suggérer des remèdes merci de bien vouloir remplir ce questionnaire bilan [crfp ; pub-pcr-1]

27) est-ce qu'on voit une forte régression de la demande [corpaix ; contrat]

28) est-ce qu'il y a une une croissance forte de-des grandes villes où il reste encore quand même une agriculture une paysannerie assez conséquente(s) [corpaix ; cameroun]

Les contraintes positionnelles de cet adjectif semblent découler prioritairement du substantif dont il dépend. Mais il faudrait déterminer les contraintes projetées par chaque substantif et les croiser avec des paramètres sémantiques pour avoir une vision exhaustive du fonctionnement de fort. Par exemple, fromage va contraindre fort à se postposer alors que odeur accepte les deux places. Là encore, de plus amples investigations seront nécessaires. Pour fort, comme pour d'autres adjectifs, il serait intéressant de mener une étude collocationnelle poussée afin d'expliquer les variations positionnelles ainsi que certains blocages observés.

Dans la partie suivante, nous proposons un classement basé exclusivement sur la place des adjectifs épithètes observée dans les corpus oraux. Notre classification repose sur la fréquence relevée en corpus de chacune des deux places possibles. Il y a d'une part les adjectifs alternants et non alternants (i.e. uniquement postposés ou antéposés). D'autre part, parmi les adjectifs alternants, nous distinguons trois cas de figure :

- Les adjectifs majoritairement antéposés ;

- Les adjectifs apparaissant autant en antéposition qu'en postposition ;

- Les adjectifs majoritairement postposés.

Chacune de ces catégories peut inclure les alternances complexes vues dans cette section. Cette classification recouvre donc des fonctionnements parfois hétérogènes. Il ne faut pas la voir comme une tentative d'uniformisation mais plutôt comme une entrée pratique basée sur un critère en partie arbitraire mais le mieux à même de montrer qu'il existe une place préférentielle pour la plupart des adjectifs en français parlé non planifié.

\section{Classement des adjectifs}

\subsection{Les adjectifs uniquement antéposés}

La majorité des adjectifs bloqués en antéposition est représentée par la classe des ordinaux (deuxième, troisième, quatrième, etc.), à l'exception notable de premier et second qui possèdent quelques occurrences en postposition (cf. section suivante). A noter que ce sont aussi les deux seuls à ne pas être formés par ajout du suffixe -ième au cardinal correspondant. Les autres adjectifs bloqués en antéposition, en plus des ordinaux, ne représentent pas une classe homogène (quelques, triple, tierce et satané), mais ils possèdent une fréquence nettement inférieure. On peut conclure qu'il existe un noyau dur d'adjectifs uniquement antéposés composé des ordinaux ( $87 \%$ des occurrences sont des ordinaux).

Contrairement aux cas ci-dessus, on trouve également des adjectifs uniquement antéposés pour lesquels on n'aurait aucun mal à trouver des occurrences en postposition à l'écrit. Il s'agit notamment de brave, pire, éternel et infime. Pour brave, par exemple, nous n'avons trouvé que des exemples tels que le suivant (avec un sens proche de « gentil ») : 
29) nous avons des coups de téléphone assez cocasses une brave dame qui nous explique qui nous a expliqué souvent /qu'ils, qu'eux/ étaient méchants avec les chats et que elle était très triste [corpaix ; Jeanne]

alors qu'il est potentiellement postposable avec un sens proche de « courageux » :

30) Plus tard, elle devait avoir son lot de malheurs. Car son père, homme brave et grand chasseur de croisés, fut pris vivant dans l'armée qui suivit la bataille de Muret et exécuté à Carcassonne [frantext; Oldenbourg]

Il semblerait toutefois qu'en français contemporain cet usage soit peu fréquent, y compris dans des variantes plus normées de la langue.

Dans cette classe, il est donc important de distinguer deux cas de figure: les adjectifs qui sont véritablement bloqués en antéposition et ceux qui ont une forte attraction pour l'antéposition. Les adjectifs de ce second type (infime, éternel, etc.) pourraient vraisemblablement passer dans la classe des adjectifs majoritairement antéposés pour peu que l'on dispose d'un corpus plus vaste. Mais il serait de toute façon préférentiellement en antéposition, comme s'il s'agissait de leur place par défaut.

Par ailleurs, il est intéressant de noter que parmi les adjectifs listés dans cette section, très peu sont monosyllabiques. Or, d'après Abeillé \& Godard (1999) notamment, plus un adjectif est court plus il a tendance à s'antéposer et plus un adjectif est long plus il a tendance à se postposer. Sans sous-estimer l'influence globale du «poids » (i.e. nombre de syllabes) sur la place de l'adjectif, ce paramètre n'est pas pertinent pour les adjectifs bloqués ou fortement corrélés à l'antéposition.

\subsection{Les adjectifs majoritairement antéposés}

Parmi les adjectifs majoritairement antéposés se trouvent les adjectifs les plus fréquents. Il s'agit dans l'ordre décroissant de petit, autre, grand, même, premier, bon, gros, beau, vieux, mauvais, meilleur, fameux, joli, haut, etc. Ces adjectifs basiques (ou élémentaires) s'antéposent à 98,5\%. Il est intéressant de regarder dans quels contextes ces adjectifs se postposent. Pour petit, par exemple, sur les 24 occurrences postposées (pour 2.644 antéposées), 21 comportent un adverbe modifieur (très, tout, plus, assez, relativement).

31) donc on a visité l'île bon c'était une île très petite [ctfp ; le voyage]

On peut donc dire que la présence d'un adverbe modifieur permet de relâcher la contrainte positionnelle.

Les trois seuls exemples sans modifieur sont pour l'un lié à une ellipse de être (et donc a une fonction d'attribut et non d'épithète) :

32) si le trou est gros et le fil petit ça tient pas [ctfp ; le cordonnier]

pour l'autre il permet vraisemblablement d'éviter l'ambiguïté avec petits-enfants (synonyme de «petitsfils $»)$ :

33) beaucoup travaillent à l'extérieur de la commune donc on les voit peu puis du fait que maintenant moi j'ai plus d'enfants petits qui vont à l'école [pfc ; 85amm1]

et le troisième est l'unique exemple où il ne semble pas y avoir de motivation particulière :

34) il faudra le mettre dans une cour petite pendant quelques jours tout seul [corpaix ; corpus3]

La postposition peut également être due au fait qu'un adjectif occupe déjà la place pré-subtantivale. Le second adjectif est alors rejeté dans la position post-substantivale :

35) à Orly il y avait une super belle bibliothèque très grande euh avec un rayon pour enfants euh très bien [corpaix ; médiathèque] 
Les deux adjectifs dans l'exemple ci-dessus peuvent permuter mais pas se postposer ou s'antéposer ensemble (sauf cas de coordination/juxtaposition). Il est important de signaler que les cooccurrences d'adjectifs se rapportant à un même substantif représentent toutefois un phénomène assez rare à l'oral non planifié où les locuteurs ont plutôt tendance à éviter ce type de tournure et à répéter le substantif (une super belle bibliothèque une très grande bibliothèque) ou bien à utiliser une relative (une super belle bibliothèque qui est très grande).

La présence d'un modifieur post-adjectival représente un autre paramètre intervenant dans la postposition :

36) les points forts sont les rôtis et les grillades euh les viandes autres que l'agneau parce que l'Aveyron est réputé pour son veau [crfp ; pro-sai-1]

La tournure autre que $X$ est particulièrement bien représentée parmi les cas de postposition rencontrés pour cet adjectif.

Comme nous l'avons vu dans la section précédente (avec l'exemple de sale), il existe également une corrélation entre la place et le sens de l'adjectif. Pour même, par exemple, la place induit clairement un changement de sens ou d'usage («identité » en antéposition vs simple mise en relief du substantif en postposition) :

37) il y a aussi une autre écriture spécifique pour la batterie pour les débutants qui est pas tout à fait du solfège c'est à peu près le même principe mais c'est avec des croix et des ronds au lieu de mettre euh des croches des doubles croches [crfp ; pri-pso-4]

38) c'est une écriture assez admirable une étude sur le fait que aujourd'hui l'association internationale de psychanalyse qui veut toujours plus de respectabilité donc de démontrable donc de scientifique donc de données objectivables euh entraîne peu à peu la psychanalyse à se séparer du principe même euh inventé par Freud qui est un principe conjectural [coralrom ; fmedst03]

Une variation de sens comparable caractérise certain, seul, propre et vrai. Dans ce cas, la différence de fréquence est directement liée à un usage plus courant d'un sens plutôt qu'un autre. On retrouve donc également des adjectifs fonctionnant selon ce principe dans la classe des majoritairement antéposés (ce qui est le cas de sale par exemple).

Dans cette partie, si les tendances statistiques ne sont guère contrastées (très large majorité en faveur de l'antéposition), il n'en reste pas moins qu'il existe des fonctionnements propres à certains couples Adjectif + Substantif. Et dans ce domaine, il est nécessaire de dresser une liste de chaque couple. Cela peut consister en des blocages (ou de fortes préférences) en postposition. Avec premier, on trouve des substantifs qui obligent l'adjectif à se postposer (en cas d'antéposition, le sens est différent) : arts premiers, équipe première, matière première, langue première... En ce qui concerne le couple monde + meilleur, monde meilleur sera préféré à meilleur monde :

39) nous pouvons commencer grâce à Dieu à construire ce monde meilleur dans lequel nos enfants et petits-enfants les vôtres comme les miens les enfants et les petits-enfants du monde entier doivent vivre et pourront vivre [coralrom ; fmedrp03]

Il peut également exister des fonctionnements spécifiques à des couples Modifieur + Adjectif. C'est le cas de plus et petit. On trouve des occurrences de ce couple aussi bien en antéposition qu'en postposition :

40) alors moi je vais pas m'amuser à avoir une cuve de pour chaque couleur euh donc on a des cuves plus petites [corpaix ; cierger]

41) et après on l'avait en T.D. et là en T.D. euh vu que c'est un plus petit groupe on savait elle savait euh nos noms et tout [pfc ; 31agc1-31alm1]

42) et dans la Chine du nord par contre c'était des des plus petits mouvements [corpaix; arts martiaux] 
Cependant, les exemples (41) et (42) sont les seuls que nous ayons trouvés en antéposition avec le modifieur plus et une autre valeur que le superlatif. Dans cette position, on retrouve le plus souvent le superlatif :

\section{3) je mets mon appareil photo sur le pied je prends la plus petite ouverture [coralrom ; ffamdl26]}

Il y aurait donc une sorte de spécialisation : antéposition lorsque l'adjectif petit est accompagné du superlatif et postposition lorsqu'il est accompagné de l'adverbe de degré. En effet, on ne trouve pas d'exemple du type l'ouverture la plus petite dans les corpus oraux que nous avons dépouillés. D'autres modifieurs et d'autres adjectifs sont sans doute concernés par ce phénomène.

Le couple tout + petit, quant à lui, est fortement corrélé à l'antéposition (contrairement aux autres modifieurs) :

44) c'est en Gambie c'est un tout petit pays dans le dans le dans le Sénégal qui fait quarante kilomètres de long [crfp ; pri-nic-1]

Pour ce qui est de l'ensemble des adjectifs majoritairement antéposés, des tendances générales se résumant à quatre paramètres permettent, au final, de rendre compte des rares cas de postposition : la présence d'un modifieur pré-adjectival ou post-adjectival, les associations privilégiées (ou collocations), les changements de sens et la présence d'un autre adjectif. Et comme nous l'avons vu, certains usages doivent faire l'objet d'une analyse extrêmement précise pour tenir compte de l'ensemble des paramètres positionnels.

\subsection{Les alternances libres}

Il existe quelques rares exemples pour lesquels la place semble totalement indifférente :

45) alors là j'ai dit je vais prendre celui-là moins lumineux parce qu'il ouvrait qu'à trois cinq alors que l'autre ouvrait à un deux c'est-à-dire il y a une différence énorme j'ai pris celui-là parce que j'avais envie de prendre en photo des surtout des insectes [coralrom ; ffamdl25]

46) bah effectivement en fait euh moi je pense qu'il y a il y a u- une énorme différence entre les deux [corpaix ; Billy]

Pour ces adjectifs, les fréquences en anté- et postposition sont très proches. Or, on n'observe pas du tout ce même équilibre dans la presse écrite, comme le montre le tableau suivant :

\begin{tabular}{|l|c|c|c|c|}
\hline \multirow{2}{*}{ Adjectifs } & \multicolumn{2}{|c|}{ Oral } & \multicolumn{2}{c|}{ Presse écrite } \\
\cline { 2 - 5 } & Antéposé & Postposé & Antéposé & Postposé \\
\hline \multirow{2}{*}{ Imorme } & $\begin{array}{c}57 \\
(46,3 \%)\end{array}$ & $\begin{array}{c}66 \\
(53,7 \%)\end{array}$ & $\begin{array}{c}102 \\
(77,7 \%)\end{array}$ & $\begin{array}{c}37 \\
(32,3 \%)\end{array}$ \\
\hline Supense & 15 & 15 & 124 & 14 \\
& $(50 \%)$ & $(50 \%)$ & $(89,9 \%)$ & $(10,1 \%)$ \\
\hline & 20 & 16 & 54 & 9 \\
$(55,6 \%)$ & $(44,4 \%)$ & $(85,7 \%)$ & $(14,3 \%)$ \\
\hline
\end{tabular}

Tableau 3. Fréquences comparées des adjectifs énorme, immense et superbe

Il semble y avoir une tendance, dans la presse écrite, à privilégier l'antéposition lorsque les deux places sont également possibles et ont une fréquence à peu près équilibrée à l'oral. C'est un indice important permettant d'illustrer le statut particulier de l'antéposition de certains adjectifs. Il s'agit sans doute d'une marque appartenant au langage soutenu. 
Pour ces adjectifs, aucune régularité particulière ne semble se dégager, excepté leur affinité avec les déterminants indéfinis et la présence d'un modifieur qui peut bloquer l'antéposition (du moins dans les exemples que nous avons observés) :

47) ça c'est toute ma base on a un groupe assez énorme [crfp ; pri-pno-1]

48) ? ça c'est toute ma base on a un assez énorme groupe

Il existe donc bien quelques adjectifs pour lesquels aucun paramètre explicatif ne peut être invoqué pour rendre compte du choix d'une place plutôt qu'une autre (hormis la présence d'un modifieur). Les locuteurs peuvent donc les utiliser en antéposition ou en postposition de manière indistincte. Pour ces adjectifs, il n'existe donc pas de place préférentielle.

\subsection{Les adjectifs majoritairement postposés}

Il existe des adjectifs très majoritairement postposés que l'on retrouve pourtant couramment antéposés dans la presse écrite. Ces adjectifs sont utilisés préférentiellement ou par défaut en postposition à l'oral non planifié et les rares exemples en antéposition se trouvent dans des transcriptions d'interventions préparées ou lues, de situations de parole surveillée : documentaires télévisés, conseils municipaux, etc. C'est le cas dans les exemples suivants :

49) enfin l'importante réduction des participations communales pour les zones d'aménagement confiées à notre SEM liées à l'achèvement de plusieurs opérations et à leurs bons résultats vient elle aussi renforcer nos moyens [crfp, pub-pno-1, conseil municipal]

50) Frodo jouit pleinement de son actuelle suprématie en l'absence de Freud [coralrom, fmedrp02, documentaire télévisé sur les chimpanzés]

L'antéposition de ces adjectifs doit donc être principalement considérée comme l'indice d'un degré élevé d'élaboration du discours, d'une situation de parole surveillée et/ou d'un écrit oralisé. Le " genre textuel » (au sens large) est également un facteur important à prendre en compte. En fait, la place de l'adjectif est fortement influencée par le «genre textuel» et ce phénomène représente donc un paramètre explicatif important.

D'autres antépositions peuvent s'expliquer par un effet du cotexte. Dans l'exemple ci-dessous, nous faisons l'hypothèse que l'occurrence de long travail dans la proximité immédiate de $d u r$ a une influence sur l'antéposition de ce dernier :

51) c'est un long travail et un dur travail [crfp, pri-tou-1]

Par un effet de mimétisme, la répétition lexicale de travail favoriserait la reproduction à l'identique de l'ordre des mots réalisé dans le premier syntagme nominal.

Par ailleurs, il arrive parfois que des adjectifs pour lesquels nous ne trouvons aucune occurrence en antéposition soient pourtant majoritairement antéposés dans la presse écrite (exemple de puissant cidessous). Nous reproduisons à la page suivante les fréquences de quelques adjectifs majoritairement (ou systématiquement) postposés en français parlé.

Il apparaît donc, comme nous l'avons dit plus haut, que dans la presse (et dans l'écrit plus généralement) il y a un nombre d'adjectifs alternants nettement supérieur par rapport à l'oral non planifié. Et pour ces adjectifs alternants supplémentaires, il ne semble pas y avoir de contrainte, préférentielle ou catégorique, permettant d'expliquer le choix d'une place plutôt qu'une autre :

52) Dernière égalité à 16/16 avec une frappe puissante de Capet, à laquelle répond illico Sapinart, tandis que Ces enfonce le clou sur une balle sauvée in extremis. [Dépêche du Midi]

53) Moukassa adressait une puissante frappe que Preau avait toutes les peines du monde à repousser (2'). [Est Républicain]

54) des élus et représentants de partis politiques dont ceux de l'actuel gouvernement [L'Humanité]

55) Chaque jour un peu mieux, les Français comprennent la brutalité du gouvernement actuel. [L'Humanité] 


\begin{tabular}{|l|c|c|c|c|}
\hline \multirow{2}{*}{ Adjectifs } & \multicolumn{2}{|c|}{ Oral } & \multicolumn{2}{c|}{ Presse écrite } \\
\cline { 2 - 5 } & Antéposé & Postposé & Antéposé & Postposé \\
\hline \multirow{2}{*}{ Puissant } & 0 & 17 & 61 & 44 \\
& $(0 \%)$ & $(100 \%)$ & $(58,1 \%)$ & $(41,2 \%)$ \\
\hline \multirow{2}{*}{ Difficile } & 0 & 98 & 15 & 154 \\
$(0 \%)$ & $(100 \%)$ & $(8,9 \%)$ & $(91,1 \%)$ \\
\hline \multirow{2}{*}{ Actuel } & 2 & 132 & 109 & 353 \\
& $(1,5 \%)$ & $(98,5 \%)$ & $(23,6 \%)$ & $(76,4 \%)$ \\
\hline \multirow{2}{*}{ Dur } & 2 & 72 & 12 & 90 \\
& $(2,7 \%)$ & $(97,3 \%)$ & $(11,8 \%)$ & $(88,2 \%)$ \\
\hline \multirow{2}{*}{ Important } & 9 & 276 & 149 & 338 \\
& $(3,2 \%)$ & $(96,8 \%)$ & $(30,6 \%)$ & $(69,4 \%)$ \\
\hline
\end{tabular}

Tableau 4. Fréquences comparées des adjectifs puissant, difficile, actuel, dur et important

Mais il faut retenir que, pour ces adjectifs, la postposition est la place utilisée spontanément à l'oral non planifié (la place "par défaut» en quelque sorte) en dehors d'un contexte nécessitant un degré d'élaboration plus important ou un niveau de langue élevé.

\subsection{Les adjectifs uniquement postposés}

Les adjectifs uniquement postposés sont de loin les plus nombreux (ils représentent plus de $90 \%$ des adjectifs). Les adjectifs les plus fréquents à cette place sont les suivants (par ordre décroissant) : français, social, général, âgé, particulier, scolaire, économique, politique, professionnel, précis, etc. Nous n'avons pas observé de phénomène particulier à leur égard. Il faut tout de même mentionner certains adjectifs pour lesquels on trouve des occurrences d'antéposition à l'écrit (cf. ci-dessus puissant et difficile) mais aucune occurrence dans les ressources orales que nous avons consultées. Comme pour la classe des adjectifs uniquement antéposés, il faut distinguer ceux qui sont véritablement bloqués et ceux pour lesquels on pourrait trouver quelques attestations moyennant un élargissement du corpus.

\section{Synthèse}

Dans les données de français parlé que nous avons observées, moins de $10 \%$ des adjectifs attestés sont alternants. Et pour une part importante des adjectifs alternants, il existe une position préférentielle. Les autres adjectifs se situent uniquement en antéposition ou en postposition. Ainsi, la plupart des adjectifs, tant en nombre d'occurrences qu'en nombre de formes :

- $\quad$ soit n'occupent qu'une seule place par rapport au substantif auquel ils se rapportent (ex : deuxième en antéposition et français en postposition);

- $\quad$ soit occupent préférentiellement une des deux places possibles (ex : grand en antéposition et important en postposition).

Lorsque les adjectifs occupent la place non préférentielle, il y a systématiquement un paramètre explicatif : présence d'un modifieur, situation de parole, degré d'élaboration du discours, etc. Plus de 95\% des adjectifs respectent le schéma décrit ci-dessus. Les adjectifs en français parlé non planifié sont donc majoritairement bloqués ou possèdent une « place préférentielle », c'est-à-dire une place sélectionnée par défaut en l'absence d'un paramètre particulier. Nous considérons que les adjectifs en distribution complémentaire (ex : prochain, sale) ont également une place préférentielle dépendant des paramètres pertinents pour chacun d'entre eux. Cela signifie donc qu'ils peuvent être tantôt antéposés tantôt postposés en fonction d'un ou plusieurs facteurs. Ainsi, la place peut être liée au sens, comme pour sale, même, seul ou certain, ou bien à des paramètres multiples comme pour prochain. Comme nous l'avons montré, ces régularités peuvent être enfreintes à l'écrit pour des raisons notamment stylistiques. 
Les quelques adjectifs restants (moins de 5\%) se partagent entre ceux qui possèdent une alternance libre (ex: immense) et ceux qui, pour l'instant, nécessitent de plus amples investigations pour en cerner précisément le fonctionnement (ex : nouveau).

En français parlé non planifié, on peut donc conclure que les deux ordres, Adjectif + Substantif et Substantif + Adjectif, ne sont pas également possibles à l'échelle du même adjectif, excepté pour une infime fraction d'entre eux, et qu'ils exhibent une régularité moins présente dans les corpus écrits que nous avons consultés. Dans les textes écrits, notamment dans la presse et la littérature, il y a une tendance à enfreindre ces régularités. C'est particulièrement visible avec puissant qui est systématiquement postposé dans le corpus oral et majoritairement antéposé dans le corpus de presse. Contrairement à une idée largement répandue, les types de textes considérés comme les plus prestigieux (parmi lesquels la littérature) ne représentent pas les supports les plus adaptés pour l'observation des régularités linguistiques, étant donné que des facteurs supplémentaires, moins présents à l'oral non planifié, vont venir «brouiller» l'observation, du moins en ce qui concerne la place de l'adjectif épithète en français. C'est l'un des intérêts de la présente description que de montrer l'importance du français parlé pour mettre en évidence les régularités fondamentales de la langue, à rebours des conceptions traditionnelles qui considèrent généralement l'oral comme une variante de la langue écrite normative remplie de scories.

\section{Références bibliographiques ${ }^{9}$}

Abeillé, A. \& Godard, D. (1999). La position de l'adjectif épithète en français : le poids des mots. Recherches linguistiques de Vincennes, $\mathrm{N}^{\circ} 28$, p. 9-32.

Benzitoun, C., Bresson, S., Budzinski, L., Debaisieux, J.-M. \& Holzheimer, K. (2010). Quand un corpus rencontre un adjectif du troisième type : Etude distributionnelle de prochain. In Oliviéri, M. (éd.), La syntaxe de corpus, Corpus, $\mathrm{N}^{\circ} 9$, p. 245-264.

Benzitoun, C. (2013). Adjectifs épithètes alternants en français parlé : premiers résultats. In Sabio, F. (éd.), TIPA, No29.

Blanche-Benveniste, C. (2010). Le français - Usages de la langue parlée. Louvain-Paris : Peeters.

Blasco-Dulbecco, M. \& Cappeau, P. (2004). Quelques remarques sur l'adjectif à l'oral. In François, J. (dir.), L'adjectif en français et à travers les langues, Caen : Presses Universitaires de Caen, Bibliothèque de Syntaxe \& sémantique, p. 413-428.

Blasco-Dulbecco, M. \& Cappeau, P. (2005). Ce que les corpus oraux nous apprennent sur les adjectifs. In Williams, G. (dir.), La linguistique de corpus, Rennes : Presses Universitaires de Rennes, p. 69-80.

Blinkenberg, A. (1928). L'ordre des mots en français moderne Première partie. Copenhague : Høst \& Søn.

Blinkenberg, A. (1933). L'ordre des mots en français moderne Deuxième partie. Copenhague : Levin \& Munksgaard.

Bouchard, D. (2002). Adjectives, Number and Interfaces: Why Languages Vary. Oxford: Elsevier Science.

Cappeau, P. (2002). Entre l'auxiliaire et le participe passé. Recherches sur le français parlé, №17, p. 11-28.

Cinque, G. (1994). On the Evidence for Partial N Movement in the Romance DP. In Cinque, G., Koster, J., Pollock, J.-Y., Rizzi, L. \& Zanuttini, R. (eds.), Paths Towards Universal Grammar, Georgetown: Georgetown University Press, p. 85-110.

Creissels, D. (2006). Syntaxe générale une introduction typologique 1 : catégories et constructions. 1/2, Paris : Hermès - Lavoisier.

Delomier, D. (1980). La place de l'adjectif en français : bilan des points de vue et théories du XX $\mathrm{X}^{\mathrm{e}}$ siècle. Cahiers de lexicologie, $\mathrm{N}^{\circ} 37 / 2$, Jacques \& Demontrond, p. 5-24.

Forsgren, M. (1978). La place de l'adjectif épithète en français contemporain Etude quantitative et sémantique. Acta Universitatis Upsaliensis, Studia Romanica Upsaliensis, 20, Uppsala.

Forsgren, M. (1997). Un classique revisité : la place de l'adjectif épithète. In Kleiber, G. \& Riegel, M. (éd.), Etudes de linguistique française, médiévale et générale offertes à Robert Martin à l'occasion de ses 60 ans, Champs linguistiques, Louvain-la-Neuve : Duculot, p. 115-126. 
Fox, G. (2012). L'acquisition des modifieurs nominaux : le cas de l'adjectif du français. Thèse de doctorat, Université Paris 3.

Goes, J. (1999). L'adjectif Entre nom et verbe. Champs linguistiques, Paris/Bruxelles : Duculot.

Knittel, M.-L. (2005). Some remarks on adjective placement in the French NP. Probus, Volume 17, Issue 2, p. 185226.

Larsson, B. (1994). La place et le sens des adjectifs épithètes de valorisation positive. Etudes romanes de Lund, 50, Lund University Press.

Noailly, M. (1999). L'adjectif en français. L'essentiel, Ophrys.

NVlke, H. (1996). Où placer l'adjectif épithète ? Focalisation et modularité. Langue Française, N¹11, p. 38-58.

Reiner, E. (1968). La place de l'adjectif épithète en français, théories traditionnelles et essais de solution. Vienne, Stuttgart, W. Braumüller.

Riegel, M., Pellat, J.-C. \& Rioul, R. (2004). Grammaire méthodique du français. Presses Universitaires de France.

Thuilier, J. (2012). Contraintes préférentielles et ordre des mots en français. Thèse de doctorat, Université ParisDiderot.

Thuilier, J. (2013). Syntaxe du français parlé vs écrit : le cas de la position de l'adjectif épithète par rapport au nom. In Sabio, F. (éd.), TIPA, No29.

Wilmet, M. (1980). Antéposition et postposition de l'épithète qualificative en français contemporain. Travaux de linguistique, $\mathrm{N}^{\circ} 7$, p. 179-201.

Wilmet, M. (1981). La place de l'épithète qualificative en français contemporain Etude grammaticale et stylistique. Revue de linguistique romane, $\mathrm{N}^{\circ} 45, \mathrm{p} .17-73$.

Wilmet, M. (1986). La détermination nominale. Paris : PUF.

Wunderli, P. (1987). La place de l'adjectif : norme et infraction à la norme. Travaux de linguistique, Nº14-15, p. 221236.

\section{Bibliographie relative aux corpus oraux utilisés}

Traitement des Corpus Oraux en Français (TCOF / http://www.cnrtl.fr/corpus/tcof/)

André, V. \& Canut, E. (2010). Mise à disposition de corpus oraux interactifs : le projet TCOF (traitement de corpus oraux en français). Pratiques, $\mathrm{N}^{\circ} 147 / 148$, p. 35-51.

\section{Corpaix}

Blanche-Benveniste, C. (1999). Constitution et utilisation d'un grand corpus. Grands corpus : diversité des objectifs, variété des approches, Revue Française de Linguistique Appliquée, №4/1, p. 65-74.

Choix de Textes en Français Parlé (CTFP)

Blanche-Benveniste, C., Rouget, C. \& Sabio, F. (2002). Choix de textes de Français parlé, 36 extraits. Paris : Champion, Collection Les français parlés.

Corpus de Français Parlé Parisien (CFPP / http://cfpp2000.univ-paris3.fr/)

Branca-Rosoff, S., Fleury, S., Lefeuvre, F. \& Pires, M. (2012). Discours sur la ville. Présentation du Corpus de Français Parlé Parisien des années 2000 (CFPP2000) - http://cfpp2000.univ-paris3.fr/CFPP2000.pdf

\section{C-ORAL-ROM}

Cresti, E. \& Moneglia, M. (2005). Integrated Reference Corpora for Spoken Romance Languages. Studies in corpus linguistics, 15, John Benjamins.

Corpus Phonologie du Français Contemporain (PFC / http://www.projet-pfc.net/) 
Durand, J., Laks, B. \& Lyche, C. (2002). La phonologie du français contemporain : usages, variétés et structure. In Pusch, C. \& Raible, W. (eds.), Romanistische Korpuslinguistik- Korpora und gesprochene Sprache/Romance Corpus Linguistics - Corpora and Spoken Language, Tübingen : Gunter Narr Verlag, p. 93-106.

Durand, J., Laks, B. \& Lyche, C. (2005). Un corpus numérisé pour la phonologie du français. In Williams, G. (éd.), La linguistique de corpus, Rennes : Presses Universitaires de Rennes, p. 205-217.

\section{Corpus de Référence du Français Parlé (CRFP)}

Equipe DELIC (2004). Présentation du 'Corpus de référence du français parlé'. Recherches sur le français parlé, $\mathrm{N}^{\circ} 18$, p. $11-42$.

\footnotetext{
${ }^{1}$ Nous tenons à remercier les relecteurs du CMLF ainsi que ceux du projet FRACOV pour leurs suggestions. Elles ont permis d'améliorer de manière substantielle la version préliminaire de cet article.

${ }^{2}$ Concernant la stylistique, « la littérature offre bien entendu le meilleur terrain d'investigation » (Wilmet, 1981: 56) pour l'étude des adjectifs.

${ }^{3}$ Les données sont celles présentes sur le site Internet du CFPP en juin 2010.

${ }^{4}$ Il s'agit uniquement des entretiens libres présents sur le site Internet de PFC en mars 2010

${ }^{5}$ Le $C E R F$ a été constitué à Aix-Marseille Université sous la direction de J. Véronis. Il s'agit d'un corpus de dix millions de mots composé de dix tranches d'un million de mots chacune. Les textes proviennent en grande partie d'aspiration de pages Internet.

${ }^{6}$ La variation diamésique porte sur les différences entre l'oral et l'écrit

${ }^{7}$ Pour une étude plus détaillée, le lecteur pourra se reporter à Benzitoun et al. (2010).

${ }^{8}$ Ce corpus écrit est extrait du CERF (voir note 5 ci-dessus). Il comporte des extraits de presse (Courrier international, Le Monde, Le Monde diplomatique, le Nouvel Observateur), des discours politiques (J. Chirac, L. Jospin, F. Mitterrand), des textes scientifiques (ouvrages publiés aux éditions du CNRS, revues parues chez Hermès, Pour la science, Sciences et avenir), des textes institutionnels (Assemblée Nationale, textes juridiques et législatifs), des textes ayant trait à la philosophie, des critiques littéraires, des critiques de films, des nouvelles de science-fiction et des romans.

${ }^{9}$ Nous avons mis en gras les références qui abordent (au moins en partie) la question de la place des adjectifs épithètes en français parlé.
} 